\title{
LIGAND BASED PHARMACOPHORE DEVELOPMENT FOR COLORECTAL CANCER DRUGS
}

1. PhD Scholar (Bioinformatics)

M.A.J.U. Islamabad.

2. Postgraduate Student (Bioinformatics) M.A.J.U. Islamabad.

3. Associate Professor Department of Bioinformatics Mohammad Ali Jinnah University (M.A.J.U) Islamabad, Pakistan.

4. Postgraduate Student (Bioinformatics) M.A.J.U. Islamabad.

\section{Correspondence Address:} Dr. Sahar fazal

Associate Professor Department of Bioinformatics Mohammad Ali Jinnah University (M.A.J.U) Islamabad, Pakistan. sahar@jinnah.edu.pk

Article received on:

24/06/2014

Accepted for publication: 25/07/2014

Received after proof reading: $16 / 10 / 2014$

\begin{abstract}
Muhammad Haseeb ${ }^{1}$, Sana Vaqar ${ }^{2}$, Dr. Sahar Fazal ${ }^{3}$, Aftab Khalil ${ }^{4}$
ABSTRACT....Objective: Focusing on the drugs used for chemotherapy and their associated side effects, there is a need to design and develop new anti-colon cancer drugs with lesser side effects and improved efficacy. Pharmacophore model proves to be a very helpful tool serving in the designing and development of new lead compounds. Place and Duration: Department of Bioinformatics, Mohammad Ali Jinnah University, Islamabad from January 2014 to May 2014. Results: In this paper, pharmacophore of 4 novel anti-colon cancer compounds has been identified and validated for the first time. Using LigandScout the pharmacophore features were predicted and 3D pharmacophore have been extracted via VMD software. A training set data was collected from literature and the proposed model was applied to the training set whereby validating and verifying their similar activity as that of the most active compounds. Conclusions: The pharmacophore model is a very handy tool for new lead compounds discovery and development. In this study pharmacophore models were built for novel drugs of colorectal cancer and they could be recommended for further studies.
\end{abstract}

Key words: $\quad$ Ligand, Pharmacophore, anti-colon cancer drugs, Computer aided drug designing, LigandScout, VMD.

Article Citation: Haseeb M, Vaqar S, Fazal S, Khalil A. Ligand based pharmacophore development for colorectal cancer drugs. Professional Med J 2014;21(5):000-000.

\section{INTRODUCTION}

Human cancer is induced by the amassment of mutations in oncogenes and tumor suppressor genes $^{1}$. Colorectal cancer (CRC) is one of the most common and recognizable types of cancer and leading causes of cancer deaths worldwide in men and women ${ }^{2}$. Colorectal cancer matures in the digestive tract, often from growths, called polyps, which are benign initially but transform into cancerous tumors. Those cancer cells permeate and damage nearby tissue, and can break away from the original tumor to form new tumors in other parts of the body through a process called metastasis.

Inherited gene mutations that increase the risk of colon cancer can be passed through families. The most common forms of inherited colon cancer syndromes are FAP and HNPCC. These rarer inherited colon cancer syndromes can be detected through genetic testing.

The current epidemiology of colorectal cancer in Pakistan shows the lower incidence in Asian countries, than in the developed countries. The rate of colorectal cancer is higher in men than in women. However a $41 \%$ rise in incidence was noted in the males between 1995 to 1999, which may indicate a higher risk in the males in future ${ }^{3}$. In men and women, it ranges from 48.3 to 72.5 and 32.3 to 56 per 100,000 per year respectively. With existing prevalence of high risk factors in Pakistan, the low CRC incidence may be a vestige. There are concerns that changing lifestyle patterns and an aging population over the next decade may translate into a higher CRC occurrence. Future screening must be considered as part of the health sector planning and include the high risk younger age groups ${ }^{4}$.

Drugs developed for cancer are single agents although for the maximum advantage they need to be used in recipe with other drugs or therapeutic agents. Initial candidate chemicals or "leads", are often recognized and tested for single agents that change cancer-cell proliferation or prolong survival. This lead to the identification of most of the clinically active cancer drugs used today. 
Specific leads then must be further optimized and assessed to characterize their pharmacokinetic and pharmacodynamic properties and evident toxic effects. Clinical evaluation is performed by trails in humans to identify a maximum tolerated dose, define severe toxic effects, and estimate bioactivity. These trails are time consuming and expensive ${ }^{5}$.

Pharmacophore is the initial step towards understanding the interaction between a receptor and a ligand. It was often postulated as the "essence" of the structure-activity knowledge gained so far ${ }^{6}$. A pharmacophore is a reasonable qualitative prediction of binding by specifying the spatial arrangement of small number atoms of function groups. The prediction can be done on large databases. Today's researcher task is to interpret the binding of anatomically varied molecules at a common receptor site. The understanding of the common properties of binding group is vital for the determination of the type of inhibitor binding the target. Pharmacophore model is very convenient for attaining this goal ${ }^{7}$.

Surface of the cell are the regions where the ligand-receptor and receptor-receptor interaction occur. The process undergo sequential levels of activity which starts initially from the cell surface and then moves towards the intracellular signaling pathways, then gene transcription which corresponds to cellular responses. Epidermal growth factor receptor (EGFR) was initially identified as an abnormally activated or mutated form which leads to a number of other abnormalities in the signaling pathway and hence leads to the formation of tumor ${ }^{8}$.

In our research, a 3D pharmacophore model was developed in order to promote the discovery of precise and effective EGFR inhibitor for the treatment of colorectal cancer. The compounds used in this study have been characterized as reported in reference papers. In order to correlate experimental and computational studies we used their bioactivity data.

\section{MATERIALS AND METHODS}

The work was initiated using LigandScout software. LigandScout is a tool for deriving the 3D from structural data of ligand complexes more speedily and evidently in a completely automated and expedient way. It offers flawless workflow both from ligand and structure based pharmacophore modeling ${ }^{9}$. LigandScout is thought to be an essential software tool for structure based drug designing, it is not only beneficial for carrying out analysis of binding sites but also for alignment based on pharmacophore and the designing of shared feature pharmacophores. LigandScout runs freely on all common operating systems. Till date a number of successful application examples have been carried out and stand published ${ }^{10}$.

The very important and the very first step in pharmacophore model generation is the selection of data set compounds. A number of drugs have been reported that are in some way related to, or used in the treatment of colorectal Cancer which include irinotecan, Regorafenib, capecitabine and some others are shown in table I.

The two dimensional (2D) chemical structures of the compounds were drawn using ChemDraw Ultra (8.0) and the structures were saved as .Pdb files. Subsequently the $2 \mathrm{D}$ structures as shown below in Fig. 1 in the form of Pdb files were imported into LigandScout and converted into corresponding 3D pharmacophore structures.

The pharmacophoric features include $\mathrm{H}$-bond donor, H-bond acceptor, Hydrophobic, aromatic, positively and negatively ionizable groups. The pharmacophore for each compound was generated and the distances among the pharmacophoric features were calculated using VMD software. VMD is designed not only for modeling, visualization, and analysis of biological systems such as proteins, nucleic acids, lipid bilayer assemblies but it may also be used to view more general molecules, as VMD can read standard Protein Data Bank (PDB) files and display the contained structure with their features. A number of application examples have been published to date ${ }^{11}$. Once the pharmacophore 


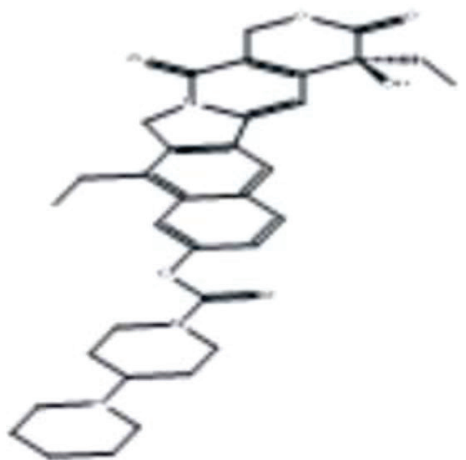

Irinotecan

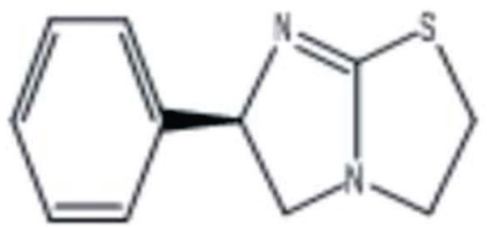

Levamisole

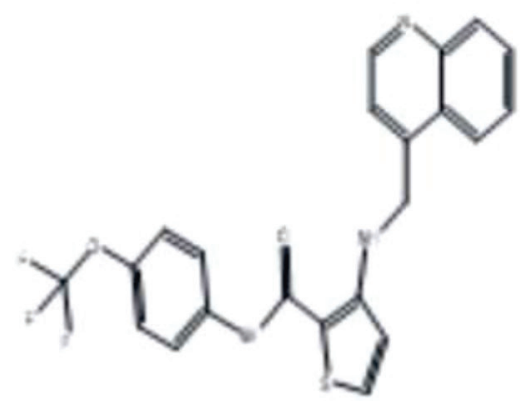

OSI-930

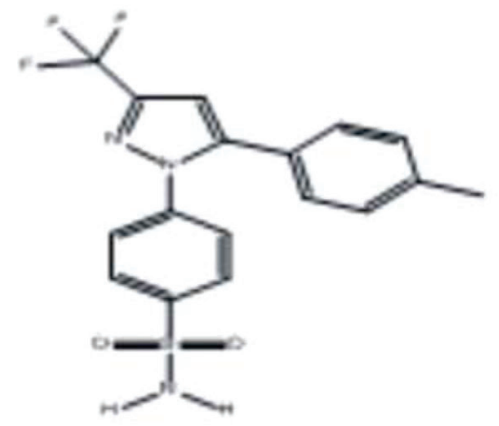

Celecoxib

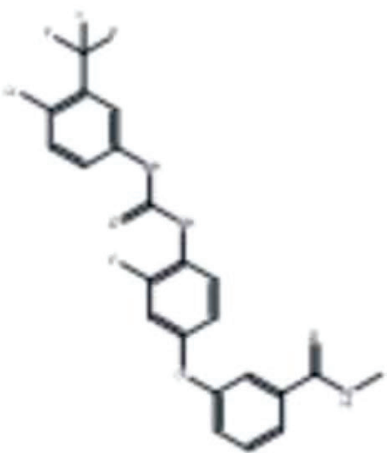

Regorafenib

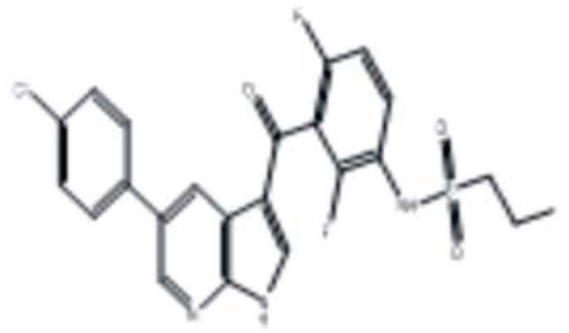

Vemurafenib

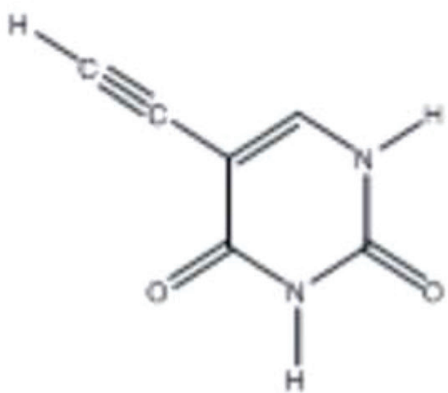

Eniluracil

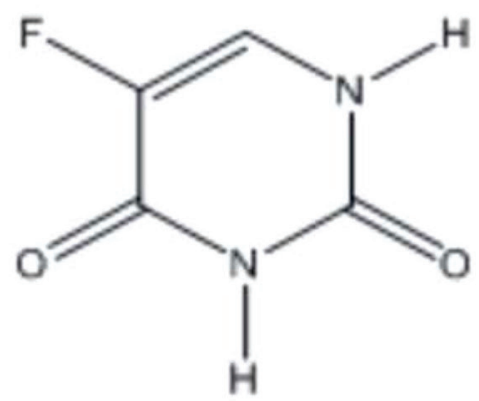

Fluorouracil

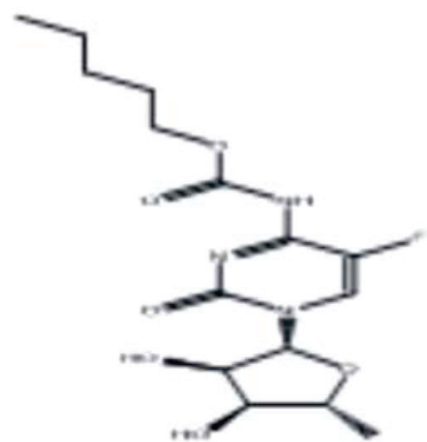

Capecitabine<smiles>CCC(C)SSc1ncc[nH]1</smiles>

PX-12

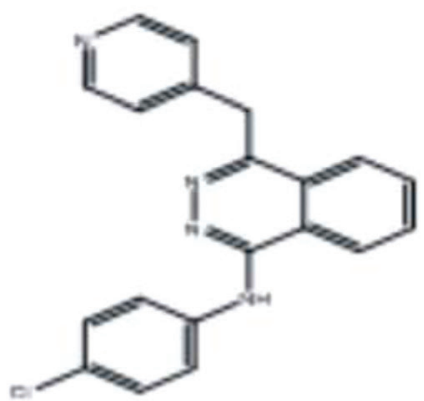

Vatalanib

Lexibulin

Fig-1 2D structures of selected data set of anti colorectal cancer 
of all the compounds were identified, the ligand was then super imposed so the pharmacophore elements overlap and a common template i-e the pharmacophore model is identified. The training set consisting of four compounds was collected from literature and it was found that the groups show enhanced and similar activity as that of the most active compounds based on the 3D pharmacophore being generated for colorectal cancer.

\section{RESULT AND DISCUSSION}

Pharmacophore analysis is considered as a fundamental part of drug design. The pharmacophore generated by LigandScout for the selected data set of anti colorectal cancer showed three main features i-e $\mathrm{H}$-bond acceptor(red vectors), $\mathrm{H}$-bond donor(white, gray vectors) and aromatic rings(yellow spheres). The representative pharacophores of each compound are shown in Fig. 2,3,4 and 5.

Resembling features were identified after analyzing the pharmacophore of all compounds generated by LigandScout. Then the similar features of all the compounds were superimposed and merged into single pharmacophore. The uniquely identified pharmacophoric features are shown in table II.

Our common featured pharmacophore predicted for four compound of anti colorectal cancer is based on one HBAs, eight HBDs and two aromatic centers. The distance triangle measured between the common pharmacophore features of each compound using VMD is shown in table 4. The distance ranges from minimum to maximum and have measured between the HBA and HBD, HBA and aromatic ring and $\mathrm{HBD}$ and aromatic ring.

The distances among the common pharmacophoric features between the predicted pharmacophore are shown in Figure 6. The distances between aromatic ring and HBD range from 3.31 to 4.5 , between aromatic rings to HBA range from 7 to 8 and between HBA to HBD range from 6 to 7 .

\section{CONCLUSIONS}

The pharmacophore model is a very handy tool for new lead compounds discovery and development. In this study, pharmacophore models were built for novel drugs of colorectal cancer, pharmacophoric features were predicted and 3D pharmacophore has been generated for colorectal cancer. A triangle of three different classes has been selected for pharmacophore and Hydrogen bond Acceptor, Hydrogen bond Donor and Hydrophobic character of standard drugs have been filtered out as key pharmacophoric feature and hence could be used for further studies. Moreover Pharmachopore based docking will be used for virtual screening and designing of some novel drugs for colorectal cancer in continuation of this work.

Copyright(C) 25 July, 2014. 


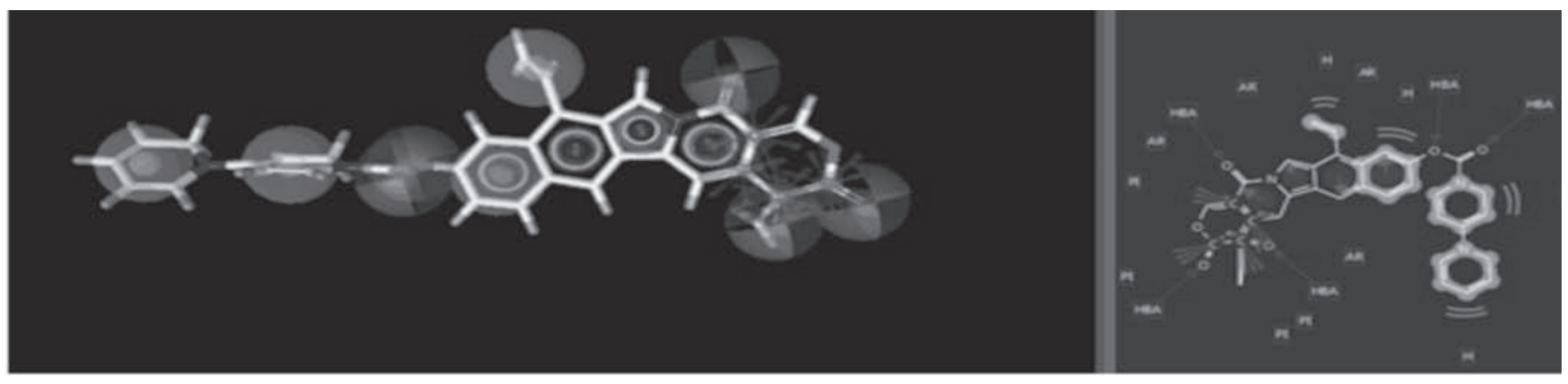

Fig-2. A pharmacophore of Irinoteean.

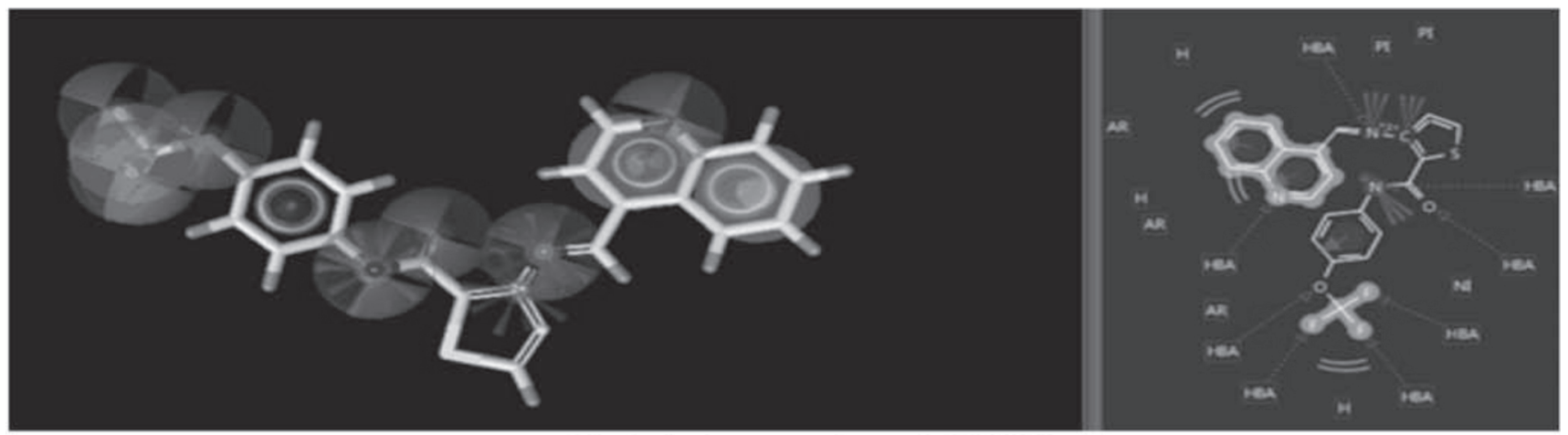

Fig-3. A pharmacophore of OSI-930

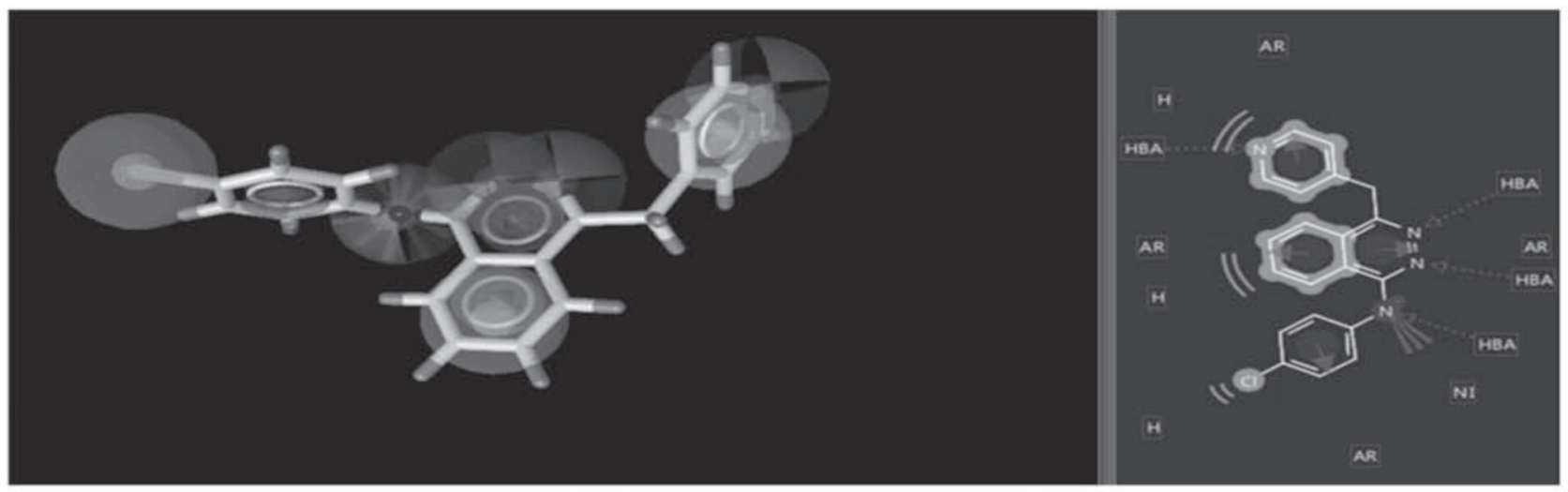

Fig-4. A pharmacophore of Vatalanib

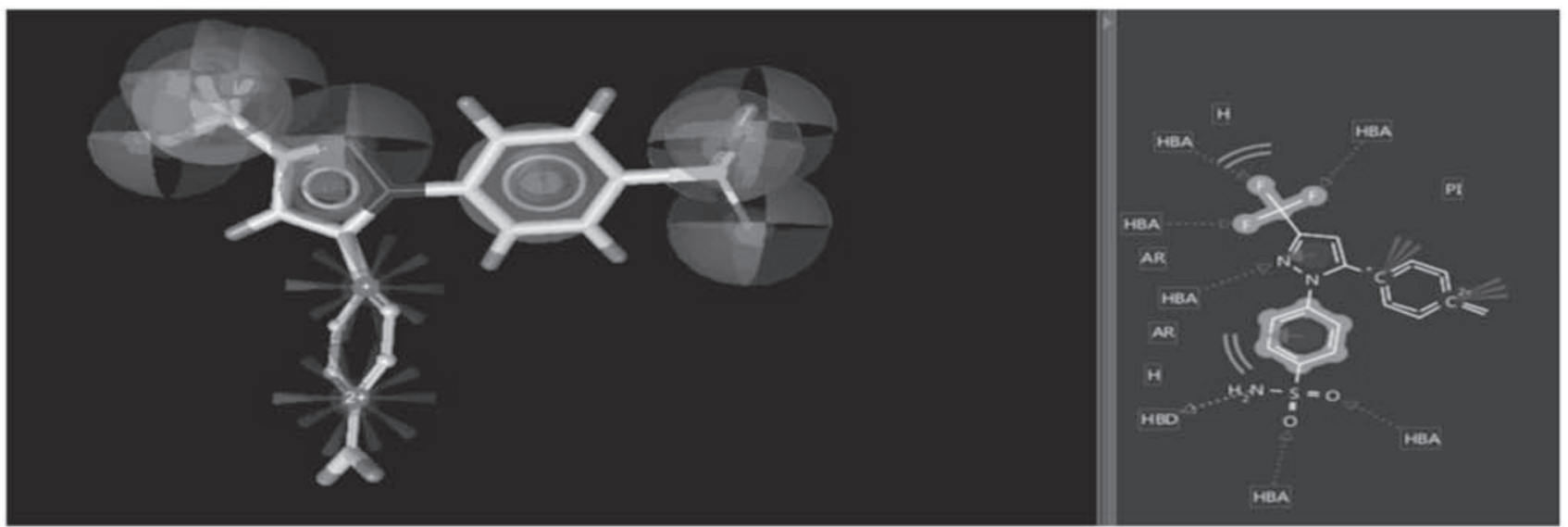

Fig-4. A pharmacophore of Celecoxib 


\begin{tabular}{|l|l|l|l|l|l|c|}
\hline S.Na & 2D Structure & $\begin{array}{c}\text { Compound } \\
\text { Name }\end{array}$ & IC50 values & HBD & HBA & $\begin{array}{c}\text { Aromatic } \\
\text { Ring }\end{array}$ \\
\hline 1 & & & & & 1 \\
& & & & & & \\
\hline 2 & & & & & \\
\hline
\end{tabular}




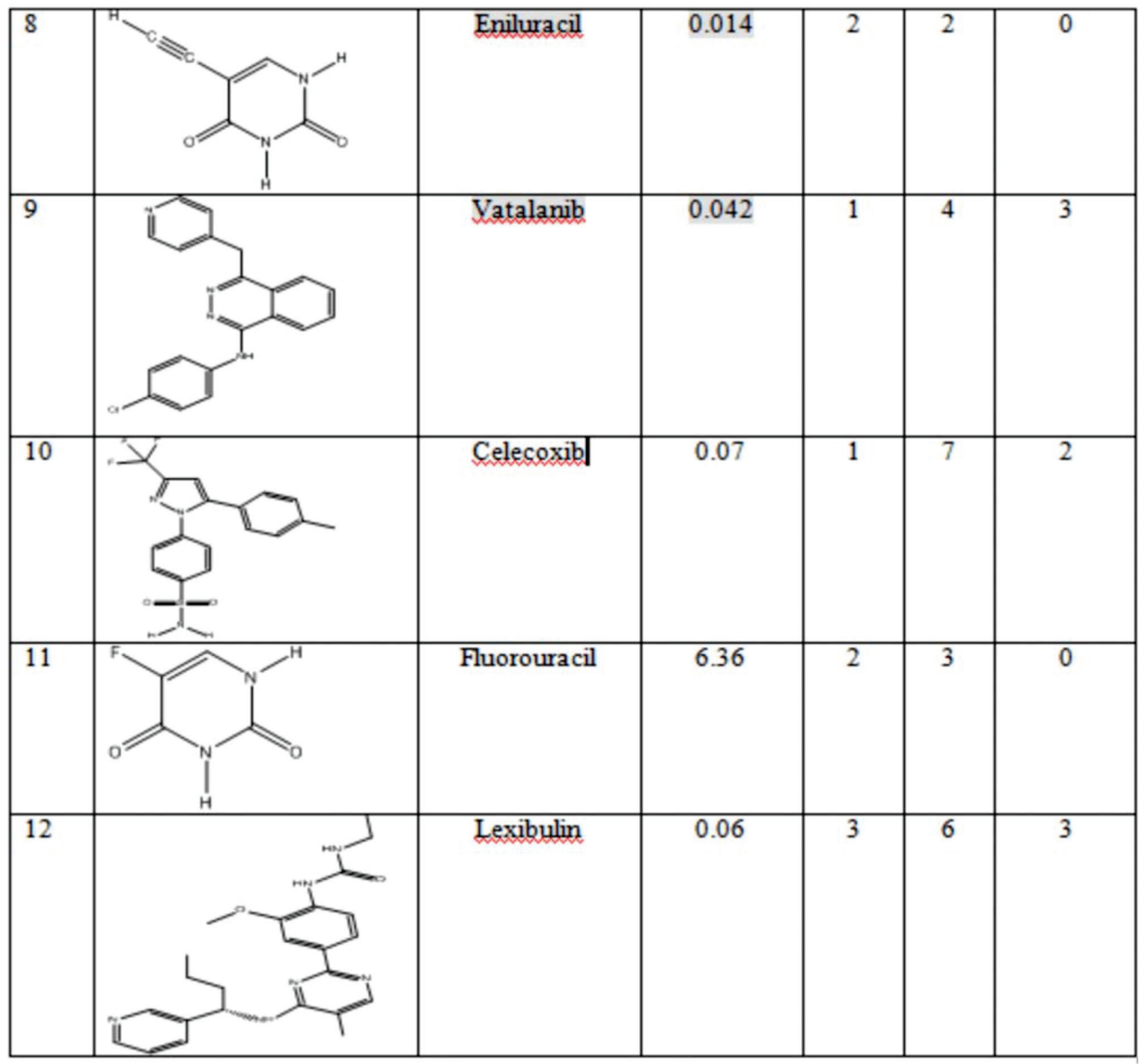

Table-I. Pharmacophoric features of each compound

\begin{tabular}{|c|c|c|c|c|}
\hline Compound Name & IC50 Value & HBD & HBA & Aromatic Ring \\
\hline Irinotecan & 0.004 & 1 & 8 & 1 \\
\hline OSI-930 & 0.005 & 2 & 8 & 2 \\
\hline Vatalanib & 0.042 & 1 & 4 & 3 \\
\hline Celecoxib & 0.07 & 1 & 7 & 2 \\
\hline
\end{tabular}




\begin{tabular}{|c|c|c|c|}
\hline Compounds & $\begin{array}{l}\text { Acceptor } \\
\rightarrow \text { Aromatic } \\
\text { Ring }\end{array}$ & $\begin{array}{l}\text { Aromatic } \\
\text { Ring } \rightarrow \\
\text { Donor }\end{array}$ & $\begin{array}{l}\text { Donor } \rightarrow \\
\text { Acceptor }\end{array}$ \\
\hline Irinotecan & $\begin{array}{l}10.95 \\
11.54 \\
10.95 \\
10.95 \\
8.87 \\
16.68 \\
11.42 \\
7.43 \\
10.84 \\
3.60 \\
9.66\end{array}$ & $\begin{array}{l}5.64 \\
9.54 \\
6.87 \\
5.81 \\
9.43 \\
10.91 \\
10.91 \\
7.39 \\
11.75 \\
4.43 \\
4.84 \\
4.74\end{array}$ & $\begin{array}{l}10.14 \\
15.85 \\
7.80 \\
14.60 \\
7.36 \\
11.64 \\
9.90 \\
11.64 \\
6.00 \\
6.00 \\
7.56 \\
9.86\end{array}$ \\
\hline OSI-930 & $\begin{array}{l}7.58 \\
8.07 \\
3.93 \\
7.51 \\
7.74 \\
7.74 \\
4.38 \\
7.89\end{array}$ & $\begin{array}{l}8.51 \\
3.32 \\
8.35 \\
5.43 \\
6.41 \\
8.45 \\
5.71 \\
9.75 \\
5.03\end{array}$ & $\begin{array}{l}6.66 \\
9.96 \\
6.87 \\
7.77 \\
5.87 \\
5.48 \\
5.74 \\
8.29 \\
5.95\end{array}$ \\
\hline Vatalanib & $\begin{array}{l}8.91 \\
8.19 \\
9.78 \\
7.63 \\
9.87\end{array}$ & $\begin{array}{l}9.48 \\
7.18 \\
3.31 \\
8.87 \\
10.27\end{array}$ & $\begin{array}{l}8.87 \\
9.48 \\
10.51 \\
6.02 \\
2.8\end{array}$ \\
\hline Celecoxib & $\begin{array}{l}4.97 \\
3.88 \\
8.44 \\
6.92 \\
8.25 \\
7.57 \\
4.75 \\
7.77 \\
4.75 \\
3.68\end{array}$ & $\begin{array}{l}3.7 \\
3.31 \\
6.62 \\
8.00 \\
3.31 \\
7.76 \\
6.93 \\
7.56 \\
7.54 \\
7.85\end{array}$ & $\begin{array}{l}2.51 \\
2.51 \\
6.37 \\
10.10 \\
9.52 \\
10.30 \\
3.28 \\
8.15 \\
6.48 \\
9.63\end{array}$ \\
\hline
\end{tabular}

\section{REFERENCES}

1. Laura D. Woodet al. The Genomic Landscapes of Human Breast and Colorectal Cancers. science 2007; $318,1108$.

2. Wenhao Weng, Junlan Feng, Huanlong Qin, Yanlei Ma. Molecular therapy of colorectal cancer: Progress and future directions. ijc 2014; 10(1002): 28722.

3. Yasmin Bhurgri et al. Pakistan - Country Profile of Cancer and Cancer Control 1995-2004. J Pak Med Assoc. 2006; 56(3):124-30.

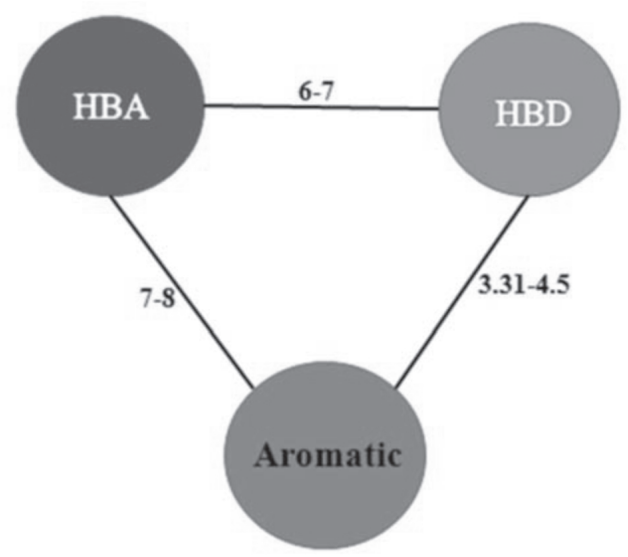

Fig-6 Distance ranges among pharmacophoric features in predicted pharmacophore

\begin{tabular}{|c|c|}
\hline Model & Training Set \\
\hline Acceptor $\rightarrow$ Aromatic Ring & $7-8$ \\
\hline Aromatic Ring $\rightarrow$ Donor & $3.31-4.5$ \\
\hline Donor $\rightarrow$ Acceptor & 6.7 \\
\hline
\end{tabular}

Table-IV. The 3D pharmacophoric distance triangle of the training set

4. Bhurgri $Y$ et al. Incidence and current trends of colorectal malignancies in an unscreened, low risk Pakistan population. 2011; 12(3):703-8.

5. Ramaswamy s. Rational Design of Cancer-Drug Combinations, N Engl J Med. 2007; 357(3):299-300.

6. Peter Gund, Evolution of the Pharmacophore Concept in Pharmaceutical Research. Pharmacopeia Inc., Princeton, New Jersey; 2007.

7. Omoshile O. Clement and Adrea Trope Mehl. HipHop: Pharmacophores based on multiple common-feature alignments. Molecular Simulation Inc. San Diego, California, 2000.

8. Mendelsohn J, Baselga J. The EGF receptor family as targets for cancer therapy. Oncogene 2000;19:6550-65.

9. Drc : A Review of LigandScout, 2008.

10. Wolber, G. Langer, T. LigandScout: 3-D Pharmacophores Derived from Protein-Bound Ligands and Their Use as Virtual Screening Filters. J. Chem. Inf. Model; 2005; 45(1); 160-169.

11. Huang, Xiaoqin, Zheng, Guangrong, Zhani, Chang-Guo. Microscopic Binding of M5 Muscarinic Acetylcholine Receptor with Antagonists by Homology Modeling, Molecular Docking, and Molecular Dynamics Simulation. journal of physical chemistry b, 2012; 116: (532-541). 